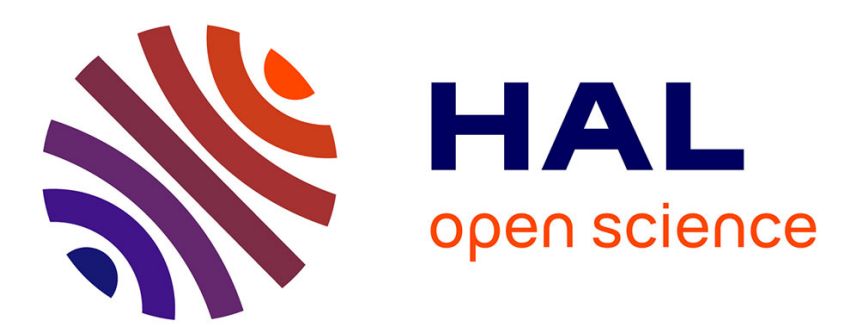

\title{
Influence du mois et de la technique de castration dans la réussite de l'haplodiploïdisation de l'orge par la méthode bulbosum
}

Pierre Devaux, Raymond Jean

\section{- To cite this version:}

Pierre Devaux, Raymond Jean. Influence du mois et de la technique de castration dans la réussite de l'haplodiploïdisation de l'orge par la méthode bulbosum. Agronomie, 1985, 5 (9), pp.795-800. hal-00884813

\section{HAL Id: hal-00884813 https://hal.science/hal-00884813}

Submitted on 1 Jan 1985

HAL is a multi-disciplinary open access archive for the deposit and dissemination of scientific research documents, whether they are published or not. The documents may come from teaching and research institutions in France or abroad, or from public or private research centers.
L'archive ouverte pluridisciplinaire HAL, est destinée au dépôt et à la diffusion de documents scientifiques de niveau recherche, publiés ou non, émanant des établissements d'enseignement et de recherche français ou étrangers, des laboratoires publics ou privés. 


\title{
Influence du mois et de la technique de castra- tion dans la réussite de l'haplodiploïdisation de l’orge par la méthode bulbosum
}

\author{
Pierre DEVAUX \& Raymond JEAN $(*)$ \\ Laboratoire de Cytologie et de Culture de Tissus, Ets Florimond-Desprez, B.P. 4I, \\ F-59242 Cappelle-en-Pévèle \\ (*) Laboratoire de Génétique écologique et de Biologie des Populations végétales, Université de Lille I, \\ F-59655 Villeneuve d'Ascq Cedex
}

Mots clés additionnels : Hordeum vulgare, H. bulbosum, morphologie d'embryons, haploìdes doublés, analyse de variance multifactorielle non orthogonale. bulbosum method.

Two factors, month and method of emasculation, were investigated in a programme of doubled haploid barley production under glasshouse conditions. 35 F3 lines of 6-rowed winter barley were crossed with Hordeum bulbosum using a pollen mixture of four clones from various sources. Crosses were done during the three months of March, April and May. Two methods of emasculation were used : 1) by clipping both lemma and palea and removing the anthers, or 2) by forming a slit with a forceps in the lemma through which the anthers are extracted. Afterwards, seed setting, seed quality and rate of embryo differentiation were recorded. Using a multifactorial non-orthogonal analysis of variance, there were significant differences between lines of barley for seed setting, for seed quality and for rates of embryo differentiation. Month had a major influence on seed setting and on embryo differentiation. The best results were obtained in May by forming a slit in the lemma for emasculation.

Additional key words : Hordeum vulgare, H. bulbosum, embryo morphology, doubled haploids, multifactorial non-orthogonal analysis of variance.

\section{INTRODUCTION}

La production d'haploïdes doublés d'orge par croisement interspécifique entre Hordeum vulgare L. et Hordeum bulbosum L. a suscité un grand intérêt auprès des sélectionneurs à cause du rendement satisfaisant de la méthode. Le protocole expérimental passe par plusieurs étapes successives au cours desquelles cependant des pertes se produisent, ce qui diminue d'autant le rendement global de la méthode. Ces pertes sont sous la dépendance, d'une part, de facteurs génétiques - certains auteurs ont en effet montré que les taux de nouaison, d'embryons différenciés, de plantes hybrides obtenues étaient influen- 
cés par le génotype des 2 parents (PICKERING \& HAYES, 1976 ; PICKERING \& MORGAN, 1979a ; SIMPSON et al., 1980 ; PICKERING, $1980 a, 1980 b$; JENSEN, 1983) - et, d'autre part, de facteurs environnementaux (JENSEN, 1976 ; Ho et al., 1978 ; PICKERING \& MORGAN, $1979 b$; PICKERING, 1980b, 1982). Dès lors, 3 démarches complémentaires sont possibles pour une utilisation plus rationnelle de la méthode :

- fabriquer des hybrides d'orge pour leur aptitude à produire des haploïdes doublés ;

- rechercher de meilleures souches d'H. bulbosum, bonnes productrices d'haploïdes ;

- parmi les multiples facteurs du milieu, définir les principaux, pour se situer dans les conditions les plus favorables possibles.

Du fait que nos descendances d'orge nous étaient imposées par le plan de sélection en cours, nous nous étions orientés, dans un travail antérieur, vers la recherche de meilleures souches d' $H$. bulbosum (DEVAuX, 1983a). Nous y avions montré l'intérèt d'un mélange de pollen des souches retenues.

La présente étude porte sur l'analyse de quelques facteurs du milieu qui interviennent dans la réussite d'un programme d'haplodiploïdisation de l'orge par la méthode bulbosum.

\section{MATÉRIEL ET MÉTHODES}

\section{A. Matériel végétal}

Le matériel orge d'hiver à 6 rangs destiné à la production d'haploïdes doublés provient d'un système complexe de croisements dans lequel figurent, parmi les géniteurs, les variétés suivantes : "Nigrate», «Dabat» et «Bosquet». Du dernier croisement, 35 descendances sont sélectionnées en F2 et conduites en F3. Les plantes de cette génération sont utilisées comme parents femelles en croisement avec $H$. bulbosum. Quatre souches d'H. bulbosum, de provenances géographiques différentes, fournissent le mélange de pollen. Elles avaient été retenues antérieurement pour leur aptitude à produire des haploïdes d'orge avec un faible taux d'hybrides (DEVAUX, 1983a).

\section{B. Les facteurs contrôlés de l'expérimentation}

Les plantes sont entrées régulièrement en serre à partir de janvier pour obtenir des floraisons échelonnées sur les mois de mars, avril et mai. Les conditions de culture en serre sont les suivantes : la température est de $20 \pm 4{ }^{\circ} \mathrm{C}$ le jour et de $16 \pm 3{ }^{\circ} \mathrm{C}$ la nuit pendant les 3 mois d'expérimentation; la photopériode de $16 \mathrm{~h}$ est maintenue si nécessaire par des lampes de type «Phytoclaude - 400 watts » donnant une densité de flux photonique au niveau des plantes de 200 $300 \mu \mathrm{E} \cdot \mathrm{m}^{-2} \cdot \mathrm{s}^{-1}$; l'humidité relative varie entre 50 et 90 p. 100 . Les plantes sont traitées contre les maladies cryptogamiques par un fongicide à base de manèbe et de thiophanate-méthyl et contre les pucerons par un insecticide à base de pyrimicarbe.

Deux techniques de castration sont expérimentées : l'une qui consiste à couper le tiers supérieur des glu- melles à l'aide de fins ciseaux et à extraire avec une pince les anthères, l'autre à réaliser une légère incision latérale dans la glumelle inférieure à l'aide d'une pince et à extraire, par cette incision, les anthères. Au moment de l'opération de castration, les 4 rangs d'épillets latéraux sont d'abord enlevés et seuls les 2 rangs médians sont conservés. On applique sur un rang médian la $1^{\text {re }}$ technique et sur l'autre, la $2^{\mathrm{e}}$. En procédant ainsi, nous évitons l'effet épi dont nous discuterons plus loin.

\section{Relevé des résultats}

Au cours de l'expérimentation sur chaque épi, nous notons successivement les 3 fréquences suivantes: le nombre de caryopses formés, de caryopses retenus pour l'extraction des embryons, d'embryons différenciés haploïdes. Précisons que les caryopses retenus pour l'extraction des embryons sont ceux qui n'apparaissent pas desséchés, car l'expérimentation nous a montré que les caryopses desséchés ne contiennent pas d'embryons aptes à se développer en culture in vitro. Lors de l'extraction des embryons, nous reconnaissons 4 types morphologiques d'embryons (fig. 1) :

- l'embryon globulaire (1),

- l'embryon cunéíforme (2),

- l'embryon haploïde (3) : il présente une gemmule - aussi l'appelons-nous différencié - et un scutellum de forme variée qui confère à l'embryon un aspect dissymétrique,

- l'embryon hybride différencié (4) reconnaissable du précédent par son axe de symétrie longitudinal.

Pour les 2 derniers types d'embryons, nous avons établi la relation caryotype-morphologie de l'embryon (DevauX, 1983b). PICKERING (1984) a confirmé cette relation. Dans nos conditions expérimentales, les embryons globulaires et cunéïformes ne se développent pas en plantes (DEVAUX, 1983a) et sont, de ce fait, écartés.

\section{Analyse des données}

A l'intérieur d'une descendance d'orge d'hiver définie, sont considérées comme répétitions les fréquences relevées sur les épis d'une même plante ou de plantes différentes (au maximum 5 plantes).

Les variables traitées par l'analyse statistique sont des proportions :

1. nombre de caryopses formés/nombre de fleurs pollinisées, proportion appelée «variable 1 »;

2. nombre de caryopses disséqués/nombre de caryopses formés, proportion appelée 《variable 2 »;

3. nombre d'embryons différenciés haploïdes/nombre de caryopses disséqués, proportion appelée « variable 3 ».

La variable 1 est équivalente au taux de nouaison.

La variable 2 mesure la qualité des caryopses.

La variable 3 est équivalente au taux d'embryons différenciés haploïdes.

Les différentes valeurs d'une variable sont réunies dans un tableau des données. Dans les tableaux des variables 1 et 2 , la variable est $Y_{i j k n}$, c'est-à-dire la 


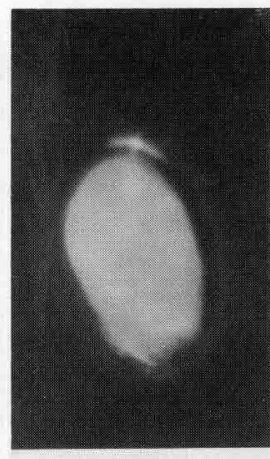

$\underline{0,25 \mathrm{~mm}}$

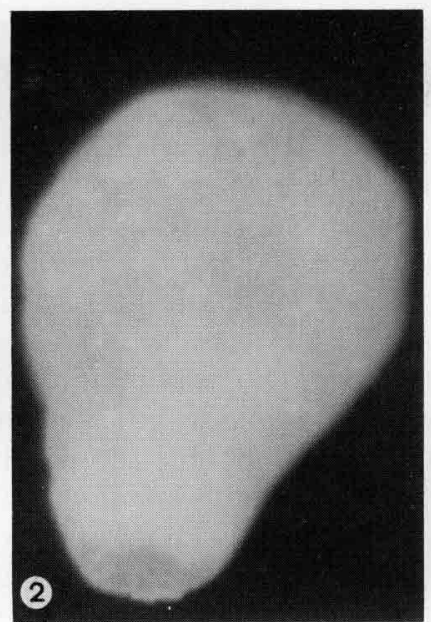

Figure 1

Morphologies d'embryons lors du prélèvement dans le caryopse, $15 j$ après la pollinisation (photos $J$. et Th. Dubois).

Embryo morphology at the time of the extraction from the seed 15 days after pollination (photos J. and Th. Dubois).

$\mathrm{n}^{\mathrm{ieme}}$ répétition de la proportion de la ligne $\mathrm{i}(\mathrm{i}=1$, $\ldots 35)$, prise au mois $\mathrm{j}(\mathrm{j}=1,2,3)$, et selon l'opération de castration $\mathrm{k}(\mathrm{k}=1,2)$. Dans le tableau de la variable 3, la variable est $\mathrm{Y}_{\mathrm{ijn}}$, car pour des raisons techniques, nous ne séparons pas les 2 techniques de castration.

Les tableaux des données des variables 1 et 2 comportent les résultats de 325 épis d'orge pollinisés par $H$. bulbosum. Comme il y a 2 opérations par épi, le nombre des relevés est de 650 . Le tableau des données de la variable 3 comporte 325 relevés, car nous ne distinguons plus les 2 techniques de castration, comme nous l'avons déjà souligné plus haut. Le nombre de répétitions par ligne, par mois et par méthode de castration varie entre 2 et 4 . Les 3 tableaux de données sont de ce fait de type non orthogonal. Ils sont traités par l'analyse de variance plurifactorielle à 3 facteurs pour les tableaux des données des variables 1 et 2 (ligne, temps, méthode de castration), à 2 facteurs pour celui de la variable 3 (ligne, temps). L'analyse est faite sur la variable exprimée en proportion et sur la variable transformée en arc sinus. L'absence d'interaction entre les 3 facteurs ayant été testée préalablement, le modèle mathématique de l'analyse de variance est additif. Les calculs ont été réalisés à l'aide de la programmathèque Amance 81 (BACHACOU et al., 1981).

\section{RÉSULTATS}

\section{A. Présentation des valeurs moyennes de l'expérimen- tation}

Les 3 tableaux des données étant trop importants, nous les résumons en présentant les moyennes exprimées en pourcentage par mois (tabl. 1) et par opération de castration (tabl. 2). Les écarts-types indiquent la distribution des moyennes obtenues par ligne d'orge d'hiver.
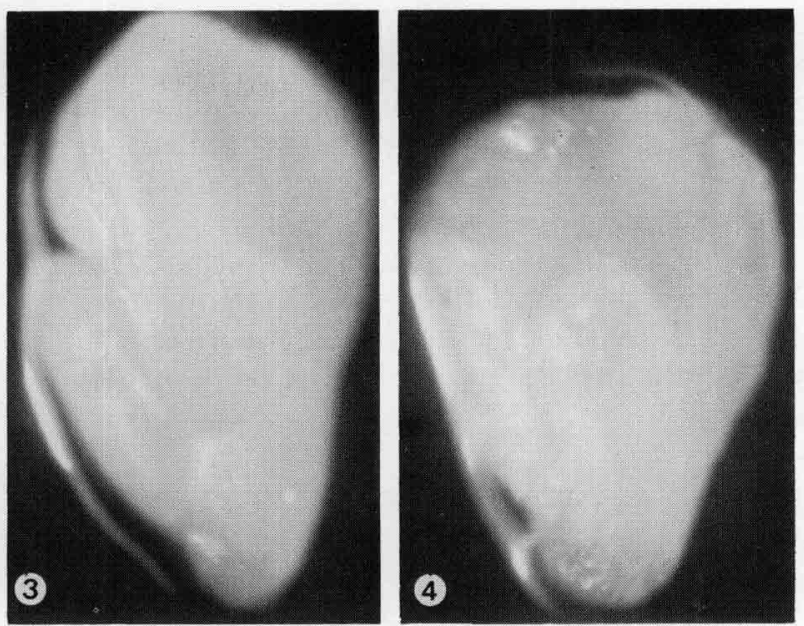

I : embryon globulaire; globular embryo;

2 : embryon cunéiforme ; wedge-shape embryo,

3 : embryon haploide différencié dissymétrique ; dissymmetrical differentiated haploid embryo;

4 : embryon hybride différencié symétrique; symmetrical differentiated hybrid embryo.

TABLEAU 1

Influence du mois sur le taux de nouaison, le taux de caryopses disséqués et le taux d'embryons différenciés. Moyenne \pm écart-type en $p$. 100, descendances 53 el techniques de castration contondues. Influence of month on rate of seed setting, dissected seeds and differentated mbryos. Mean \pm standard deviation as \%, 3 lines and enlasculation methods confounded.

\begin{tabular}{cccc}
\hline $\begin{array}{c}\text { Variable } \\
\text { analysée }\left(^{*}\right)\end{array}$ & Mars & Avril & Mai \\
\hline T1 & $60,4 \pm 7,8$ & $68,6 \pm 0,3$ & $75,0 \pm 7,0$ \\
T2 & $54,0 \pm 1,9$ & $57,4 \pm 1,4$ & $56,2 \pm 2,1$ \\
T3 & $19,3 \pm 5,0$ & $25,8 \pm 1,8$ & $29,8 \pm 5,5$ \\
\hline \hline
\end{tabular}

(*) Variables T1 : Taux de caryopses formés par rapport au nombre de fleurs pollinisées. Rate of seed setting in relation to the number of pollinated flowers.

T2 : Taux de caryopses disséqués par rapport au nombre de caryopses formés. Rate of dissected seeds in relation to seeds set.

T3 : Taux d'embryons différencies haploides par rapport au nombre de caryopses disséqués. Rate of differentiated haploid embryos in relation to the number of dissected seeds.

TABLEAU 2

Influence de la technique de castration sur le taux de nouaison et le taux de caryopses disséqués. Moyenne \pm écart-type en p. 100, descendances $F 3$ et mois confondus.

Influence of emasculation method on rate of seed setting and dissected seeds. Mean \pm standard deviation as \% 13 lines and monihs confounded.

\begin{tabular}{ccc}
$\begin{array}{c}\text { Variable } \\
\text { analysée }\left(^{*}\right)\end{array}$ & $\begin{array}{c}\text { Méthode de castration } \\
\text { Glumelles } \\
\text { coupées }\end{array}$ & $\begin{array}{c}\text { Incision latérale } \\
\text { dans la lemme }\end{array}$ \\
\hline T1 & $65,1 \pm 3,1$ & $71,4 \pm 3,1$ \\
T2 & $54,6 \pm 1,4$ & $57,4 \pm 1,4$ \\
\hline \hline
\end{tabular}

(*) Mêmes variables 1 et 2 que dans le tableau 1. Same variables 1 and 2 as in table 1 . 
Une analyse de visu du tableau 1 montre une progression nette des taux de nouaison et des taux d'embryons différenciés haploïdes en fonction de l'avancée vers les mois d'été. La progression n'est pas nette pour les pourcentages des caryopses disséqués par rapport aux caryopses formés.

En ce qui concerne l'opération de castration (tabl. 2), l'extraction des anthères par l'intermédiaire d'une fente réalisée dans la glumelle inférieure semble favoriser la réussite de l'expérimentation pour les 2 variables.

\section{B. Analyse de variance multifactorielle non ortho- gonale}

Les valeurs des coefficients $F$ calculées sur la variable proportion sont pratiquement identiques à celles calculées sur la variable transformée en arc sinus (exemple : pour la variable 1 et l'effet ligne, $F$ est égal à 2,28 avec la variable proportion, il est égal à 1,93 avec la variable transformée). Dans le tableau 3, nous portons les valeurs de $F$ calculées sur les proportions.

L'analyse de variance révèle d'abord un effet ligne d'orge d'hiver pour les 3 variables, significatif à 1 p. 1000 pour le taux de nouaison et à 1 p. 100 pour les taux d'embryons différenciés. Par contre, cet effet n'apparaît pas pour la qualité des caryopses. L'opération de castration présente un effet significatif à 1 p. 1000 sur le taux de nouaison qui est ensuite atténué à l'étape ultérieure.

\section{DISCUSSION}

Avant de dégager les aspects pratiques de notre expérimentation, nous devons d'abord justifier le choix des 2 techniques de castration et leur application simultanée au même épi. Deux autres techniques auraient pu être utilisées mais nous les avons éliminées pour des raisons pratiques. La $1^{\text {re }}$, celle qui blesse le moins la fleur, consiste à écarter lemma et palea avec une pince fine et à saisir ensuite les 3 anthères. Elle présente comme avantage de limiter l'assèchement du pistil mais elle est d'une pratique plus difficile sur des plantes de serre. En effet, sur ces dernières, l'anthèse a lieu lorsque l'épi est encore enveloppé dans la gaine. A ce stade, les tissus des glumelles sont mous et leur écartement demande, à notre avis, un temps trop long pour une technique de routine.

\section{TABLEAU 3}

Valeurs des coefficients $F$ de l'analyse de variance. $F$ values of the analysis of variance.

\begin{tabular}{cccc}
\hline \hline $\begin{array}{c}\text { Variable } \\
\text { analysée }\left(^{*}\right)\end{array}$ & Ligne & Mois & $\begin{array}{c}\text { Opération } \\
\text { castration }\end{array}$ \\
\hline T1 & $2,28^{* *}$ & $20,28^{* * *}$ & $12,01^{* * *}$ \\
T2 & $4,18^{* *}$ & 0,35 N.S. & 0,94 N.S. \\
T3 & $1,75^{* *}$ & $6,61^{* *}$ & \\
\hline \hline
\end{tabular}

${ }^{*}$ ) : Mêmes variables que dans le tableau 1 . Same variables as in table 1 .

$\left.{ }^{* *}\right): F$ significatif au seuil de 0,01 . Significant $F$ at 0.01 level. $\left({ }^{* * *}\right)$ : $\mathrm{F}$ significatif au seuil de 0,001 . Significant $\mathrm{F}$ at 0.001 level.
La $2^{e}$, proposée par DAHIYA \& JATASRA (1979), consiste à sectionner transversalement la fleur à un stade où les anthères sont immatures, sans toucher le pistil. Cette pratique est évidemment rapide mais elle lèse fortement la fleur, elle favorise sa dessiccation et nous avons constaté que la croissance ultérieure était affectée. De ce fait, nous ne l'avons pas utilisée.

Les 2 techniques de castration que nous avons retenues nous paraissent faciles à adapter à un programme de production d'haploïdes. Nous avons ensuite appliqué les 2 techniques de castration à un même épi. Il faut reconnaître qu'une telle démarche expérimentale présente à la fois un inconvénient et un avantage. L'inconvénient est d'introduire dans la comparaison l'effet de la compétition physiologique entre des épillets traités de manière différente (L. JESTIN, comm. pers., 1985). L'avantage est de pouvoir comparer les 2 techniques de castration sur le même épi ; car, si celles-ci étaient appliquées à des épis différents, nous introduirions dans la comparaison « l'effet épi ». Cette réaction propre à l'épi est connue des auteurs : pour tester l'incompatibilité dans le croisement $H$. vulgare par $H$. bulbosum, PICKERING (1979) réalise 2 croisements sur le même épi pour comparer les génotypes de pollen. De BUYSER \& HENRY (1979) et PICARD (1984) constatent un rendement différent de l'androgenèse en fonction de l'épi. Ces derniers auteurs mettent en évidence l'effet épi et le premier a voulu éliminer cet effet dans son expérimentation. Nous-mêmes, nous avons tenu compte de l'expérience de ces auteurs pensant que l'effet épi devait être contourné dans l'expérimentation.

Le but de notre travail était d'améliorer le rendement en plantes haploïdes en analysant quelques facteurs du milieu des $1^{\text {res }}$ étapes de la méthode bulbosum. Nous avons recherché un plus fort taux de nouaison ainsi qu'une meilleure qualité de caryopses et d'embryons pour les manipulations ultérieures de la méthode. Parmi celles-ci, figurent le développement in vitro des embryons différenciés et le repiquage de la jeune plante en terre. Nous avons constaté que les embryons différenciés obtenus durant les mois d'hiver sont de plus petite taille et sont moins aptes à se développer in vitro que ceux obtenus durant les mois de printemps et d'été. Dans le cadre de notre expérimentation, réalisée pendant les 3 mois de printemps, ces différences sont moins importantes. En outre, la perte des plantes après transfert en terre est, dans nos conditions de serre, négligeable.

Les facteurs les plus favorables pour l'expérimentateur sont finalement les suivants :

$1^{\circ}$ Lors de l'opération de castration, les étamines doivent être extraites par l'intermédiaire d'une fente latérale réalisée dans la glumelle inférieure et non pas après avoir coupé la partie terminale des glumelles.

$2^{\circ}$ La période de l'année la plus favorable en serre est le mois de mai avec des résultats encore satisfaisants en avril et juin.

$\mathrm{Vu}$ le grand nombre de génotypes testés par ces 2 procédés, nous pouvons affirmer qu'ils ont une application générale chez $H$. vulgare. Mais en conseillant à l'expérimentateur cette méthode de travail, nous sommes conscients que nous lui imposons des contraintes pour le déroulement complet de la méthode bulbosum car les croisements étant faits en 
mai, les jeunes plantes haploïdes sont transférées en terre en juin-juillet, période la plus défavorable au tallage. Suivant l'origine des parents $H$. vulgare, certaines plantes haploïdes donnent un nombre de talles satisfaisant pour être traitées à la colchicine mais d'autres donnent uniquement la talle principale qui monte en inflorescence et pour lesquelles il sera plus difficile d'obtenir la diploïdisation. Pourtant à cause de leur valeur génétique, nous conservons ces dernières et essayons de les faire taller après traitement à la colchicine et vernalisation en supprimant la dominance apicale et en fournissant un bon apport azoté. La $2^{\mathrm{e}}$ contrainte culturale est la période de floraison durant les mois d'automne et d'hiver pendant lesquels il faut assurer une photopériode et une thermopériode ajustées.
Ces contraintes, bien que gênantes, n'enlèvent pas du tout à la méthode que nous préconisons son intérêt car il est essentiel, au départ, d'avoir un grand nombre de caryopses et d'embryons haploïdes de bonne qualité tout en acceptant d'aller à contre-saison pour l'élevage des plantes haploïdes.

Reçu le 13 novembre 1984. Accepté le 10 mai 1985.

\section{REMERCIEMENTS}

Cette étude a été réalisée aux Etablissements de sélection Florimond-Desprez à Cappelle-en-Pévèle (Nord) et au Laboratoire de Génétique écologique et de Biologie des Populations végétales de l'Université de Lille I. Le calcul statistique a été effectué avec l'aide de M. Caille et J. P. Masson du Laboratoire de Biométrie, I.N.R.A., Rennes.

\section{RÉFÉRENCES BIBLIOGRAPHIQUES}

Bachacou J., Masson J. P., Millier C., 1981. Manuel de la programmathèque Amance 81, Dép. Biom., I.N.R.A., 516 p.

Dahiya B. N., Jatasra D. S., 1979. A rapid method of handcrossing barley. Indian J. Agric. Sci., 49, 915-917.

De Buyser J., Henry Y., 1979. Androgenèse sur des blés tendres en cours de sélection. 1. - L'obtention des plantes in vitro. Z. Pflanzenzüchtg., 83, 49-56.

Devaux P.. 1983a. Haploïdes doublés issus d'orges d'hiver par la méthode bulbosum. Analyse cytogénétique et recherche de meilleures souches $d^{\prime}$ Hordeum bulbosum. Thèse $3^{\mathrm{e}}$ Cycle, Univ. Sciences et Techniques, Lille, $125 \mathrm{p}$.

Devaux P., 1983b. Analyse cytogénétique de l'hybride Hordeum vulgare $\times H$. bulbosum et de cytotypes dérivés. Bull. Soc. Bot. $N$. Fr., 36, 61-70.

Ho K. M., Stokkermans N. J., Sadler B. J., McIntosh K. C., Ho L. C., 1978. Efficiency of barley haploid production. Barley Genet. Newsl., 8, 53-55.

Jensen C. J., 1976. Barley monoploids and doubled monoploids : techniques and experience, 316-345. In : Gaul H., Barley genetics III. Proc. 3 rd Int. Barley Genet. Symp., Garching, 1975, R.F.A., $849 \mathrm{p}$.

Jensen C. J., 1983. Producing haploid plants by chromosome elimination. In : Cell and tissue culture techniques for cereal crop improvement, Science Press, Beijing, China 1983, $55-79$ (Gordon \& Breach Sci. Publ., Inc. N.Y., 1983)

Picard E., 1984. Contribution à l'étude de l'hérédité et de l'utilisation en sélection de l'haplodiploidisation par androgenèse in vitro chez une céréale autogame: Triticum aestivum $L$. Thèse Doct. d'Etat, Univ. Paris XI, 269 p.
Pickering R.A., 1979. Further investigations on partial incompatibility in crosses between Hordeum vulgare L. and $H$. bulbosum L. In : Zeven A. C. \& Van Harten A. M., 1978, Proc. Conf. Broadening Genet. Base Crops, p. 319-325, Wageningen, Pays-Bas.

Pickering R. A., 1980a. Use of the doubled haploid technique in barley breeding at the Welsh Plant Breeding Station. Rep. Welsh Plant Breed. Stn for 1979, 208-226.

Pickering R. A., 1980b. Attempts to overcome partial incompatibility between Hordeum vulgare L. and $H$. bulbosum L. Euphytica, 29, 369-377.

Pickering R. A., 1982. The effect of pollinisation bag type on seed quality and size in Hordeum inter-and intraspecific hybridization. Euphytica, 31, 439-449.

Pickering R. A., 1984. The influence of genotype and environment on chromosome elimination in crosses between Hordeum vulgare $\mathrm{L}$. $\times$ Hordeum bulbosum L. Plant Sci. Lett., 34, 153-164.

Pickering R. A., Hayes J. D., 1976. Partial incompatibility in crosses between Hordeum vulgare L. and H. bulbosum L. Euphytica, 25, 671-678.

Pickering R. A., Morgan P. W., 1979a. Production of doubled haploid barley. Rep. Welsh Plant Breed. Stn for 1978, 84-85.

Pickering R. A., Morgan P. W., 1979b. Progress in doubled haploid production at the Welsh Plant Breeding Station. Barley Newsl., 22, 141-143.

Simpson E., Snape J. W., Finch R. A., 1980. Variation between Hordeum bulbosum genotypes in their ability to produce haploids of barley, Hordeum vulgare. Z. Pflanzenzüchtg., 85, 205-211. 